\title{
Contribution of haptic information to the process by which a mental image of ownership enhances value
}

Kenji Hirose $^{1^{*}}$, Takafumi Sasaoka ${ }^{1}$, Kentaro Ono $^{1}$, and Shigeto Yamawaki ${ }^{1}$

${ }^{1}$ Hiroshima University, Center for Brain, Mind and KANSEI Sciences Research, Hiroshima City, 7348551, Japan

*Corresponding author: hiroseken@hiroshima-u.ac.jp

\begin{abstract}
It is known that the value of an object can be increased by forming an image of owning the object

without actually possessing it. However, the detailed mechanism behind this phenomenon remains

unknown. In this study, we conducted two experiments using newly created three-dimensional

abstract objects to examine whether the formation of a mental image of ownership increases the value

of an object when a participant has a haptic experience of the object. The results suggest that when

participants' mental visual imagery ability was relatively high, having a haptic experience increased

the value of the object through their image of ownership. For others, however, imagining ownership

lowered the value of the object. The results also indicate that obtaining spatial information about an

object through a haptic experience increases the value of the object. This study suggests that haptic

information about an object contributes to the mechanism modulating how the value of an object is

influenced by individual differences in visuospatial imagery ability.
\end{abstract}




\section{Introduction}

It is important in various fields such as economic activities to clarify the mechanism by which humans recognise the value of an object and its changes. The major indicators of value include how much money one is willing to pay for the object (willingness to pay) and how much one likes the object (preference). An example of a change in value is an increase in preference due to the ownership of an object ${ }^{[1]}$. Owning an object not only increases its value but also changes one's perceptions and memories about and attention to it (e.g. ${ }^{[2-4]}$ ). In this regard, it is important to clarify the mechanism of an increase in an object's value by ownership, not only in terms of its application to practical situations such as economic activities, but also in terms of its potential to contribute to the clarification of various psychological changes that occur simultaneously with the increase in value.

However, the underlying mechanisms behind the enhancement of value remain unclear.

It is known that the preference for an object may be increased by imagining owning the object, without the actual ownership of the object. Kim and Johnson suggested that the preference for an everyday object (e.g. an electrical appliance) increases with imagining a situation in which one owns it (e.g. receiving a gift or buying something, as noted in their discussion) ${ }^{[5]}$. At the same time, it has been shown that the preference for an object does not increase when one imagines that others own it.

The influence of mental image, as described above, can be interpreted as a mere ownership effect $^{[1]}$. The explanation for the mere ownership effect is motivational in nature: to satisfy the desire 
to see oneself in a favourable light, people will overvalue an object associated with the self, namely, an owned object (e.g. self-enhancing bias ${ }^{[1]}$. Although the mechanism by which an object becomes associated with the self remains unclear, some findings suggest details about this mechanism.

In a study by Peck and Shu, when participants were asked to generate an image of owning a Slinky and a mug, both the psychological ownership (the feeling that something is one's own, regardless of actual/legal ownership ${ }^{[6]}$ ) and the willingness to pay for those goods increased ${ }^{[7]}$. Although Kim and Johnson's study did not specifically measure psychological ownership, participants were asked to form an image of ownership, so it is reasonable to assume that psychological ownership also increased $d^{[5]}$.

In addition, Iseki and Kitagami found that imagining oneself touching everyday objects and thinking about how they feel (image of touching) increased not only psychological ownership of the objects but also the sense of physical control (the feeling of having or being able to manipulate an object by oneself $\mathrm{f}^{[8]}$ ). The structural equation modelling analysis suggests that the image of touching improves one's sense of physical control over an object, and this sense of physical control increases psychological ownership, which in turn increases willingness to pay (i.e. the value of the object ${ }^{[8]}$. A similar result was obtained in a study by Peck, Barger, and Web ${ }^{[9]}$.

Therefore, one of the reasons why the image of ownership causes the object to be associated with oneself and consequently makes it feel more valuable, is presumably because it improves the sense of physical control of the object. If the sense of physical control is improved by the image of ownership, 
it is noteworthy to ask why this is so. The sense of physical control of actual objects is, of course, improved by manipulation. In order to manipulate an object, we need to touch it; and when we do touch it, we receive haptic information about the object. In the study by Iseki and Kitagami, we can assume that the sense of physical control was improved by forming the image of touching because the sense of physical control was reproduced as if one were manipulating the object by recalling haptic information ${ }^{[8]}$. Similarly, when the image of owning an object is formed, the haptic information of the object should be recalled, which reproduces the sensation of the object being manipulated, improving the sense of physical control and increasing the value.

In the present study, we examined whether the presence or absence of the haptic experience of an object influences the increase of the object's value through the image of owning it. Specifically, participants were asked to perform an association task in which they imagined themselves or others owning an object, and to rate their preference for the object before and after the task. In Experiment 1, participants were asked to touch an object to provide haptic information. In Experiment 2, participants did not touch the object. In Experiment 1, it was hypothesised that the value of the object would increase when it was associated with the self, but not when associated with others. In Experiment 2, however, value was not expected to increase in either condition.

It is important to note that using concrete objects that we see every day and know well (e.g. an electrical appliance, as used in the study by Kim \& Johnson ${ }^{[5]}$ ) can be problematic. This is because such objects are accompanied by tremendous information that we possess as existing knowledge, in 
addition to the information obtained through haptic grasping, and there is a possibility that such

information will be recalled when we form an image of ownership. Thus, even if the object's value is improved, it is impossible to distinguish whether it is due to haptic or other information. To overcome this problem of participants' existing knowledge, we used novel objects (blocks, Figure 1) as stimuli in this study.



Figure 1: An example of the blocks used in this study

A scene of owning something could be, for example, receiving a gift or making a purchase (as discussed by Kim and Johnson ${ }^{[5]}$ ). The mental image of such a scene contains many visuospatial elements. Studies have shown that there are individual differences in visuospatial imagery abilities 
(e.g. $\left.{ }^{[10,11]}\right)$. Therefore, it is necessary to consider individual differences in visual imagery ability regarding the effect of the image of ownership on block preference.

In this study, we used the Visual Imagery Style Questionnaire (VISQ ${ }^{[12]}$, which is based on the Object-Spatial Imagery Questionnaire (OSIQ) $)^{[13]}$, as a measure of visual imagery ability to classify participants into high and low imagery ability groups. Similar to the OSIQ, the VISQ individually measures the extent to which people use mental imagery of visual attributes such as shape, size, colour, and brightness (object imagery), and mental imagery of spatial attributes such as spatial relationships and arrangements between parts of objects (structure) and the movement and change of objects (spatial imagery) in their daily lives. Several questions pertain to imagery abilities, such as 'When I close my eyes, I can easily recall scenes I experienced in the past' (object imagery) and 'I can easily visualise and rotate three-dimensional figures in my mind' (spatial imagery). Therefore, the sum of the VISQ scores may be a useful indicator of overall visual imagery ability. By using the VISQ, the present study is expected to clarify how each object and spatial imagery ability, as well as the overall visual imagery ability based on the total score, contributes to the increase in the objects' value.

\section{Experiment 1}

\section{Results}

In this experiment, participants were asked to imagine a situation in which they or someone else 
owned a block, and then rate their preference for the object before and after the association task (see Method for details). To examine whether and how overall visual imagery ability affects the impact of the image of ownership, a 2 (Group: High or Low total VISQ [hereafter abbreviated as 'High/Low total group'; 11 and 14 participants, respectively]) $\times 2$ (Assignment: Self [participants] or Other [experimenter] $) \times 2$ (Time of rating: Pre or Post [association task]) repeated-measures analysis of variance (ANOVA) on average preference ratings was conducted. The results revealed a significant main effect of Time of rating $\left(F(1,23)=9.227, p=0.006, \eta_{\mathrm{p}}{ }^{2}=0.286\right)$. The average preference ratings were higher for Post than Pre rating. The three-way interaction $(F(1,23)=7.340, p=0.013$, $\eta_{\mathrm{p}}^{2}=.242$ ) was also significant (Figure 2).



Figure 2: Mean values of the Pre and Post preference ratings in the Self and Other conditions for High and Low total groups (Experiment 1). ${ }^{*} p<0.05,{ }^{* * *} p<0.005$. Error bars show 
standard error.

The three-way interaction was broken down into simple effects, which showed a significant simple interaction of Group $\times$ Time of rating on Other condition $\left(F(1,46)=6.254, p=0.016, \eta_{\mathrm{p}}{ }^{2}=0.120\right)$. The simple interaction of Assignment $\times$ Time of rating on the High total group was also significant $(F$ $\left.(1,23)=5.096, p=0.034, \eta_{\mathrm{p}}^{2}=0.181\right)$

Further analyses for simple main effects revealed that in the Low total group, the Post rating for the Other condition was significantly higher than the Pre rating $(F(1,46)=11.873, p=0.001, \mathrm{~d}=1.029)$. In the High total group, the Post rating for the Self condition was significantly higher than the Pre rating $(F(1,46)=4.841, p=0.033, \mathrm{~d}=0.720)$. These results indicate that the average preference ratings were higher in the Post than Pre rating for the Other and Self conditions of the Low and High total groups, respectively.

Next, to examine whether and how spatial-visual imagery ability affects the impacts of the image of ownership, a 2 (Group: High or Low spatial VISQ [hereafter 'High/Low spatial group'; 14 and 15 participants, respectively] $) \times 2$ (Assignment: Self or Other $) \times 2$ (Time of rating: Pre or Post) repeatedmeasures ANOVA was conducted. There was a significant main effect of Time of rating $(F(1,27)=$ 15.058, $p=0.001, \eta_{\mathrm{p}}{ }^{2}=0.358$ ). The average preference rating was higher for Post than Pre rating, and there was a significant interaction of Group $\times$ Time of rating $\left(F(1,27)=4.433, p=0.045, \eta_{\mathrm{p}}{ }^{2}=0.141\right.$; Figure 3). The results of multiple comparisons showed significant difference between the Pre and Post ratings in the Low spatial group $\left(F(1,27)=17.916, p=0.0002, \eta_{\mathrm{p}}{ }^{2}=0.399\right)$. This indicates that the 
average preference rating was higher in the Post than Pre rating in the Low spatial group.

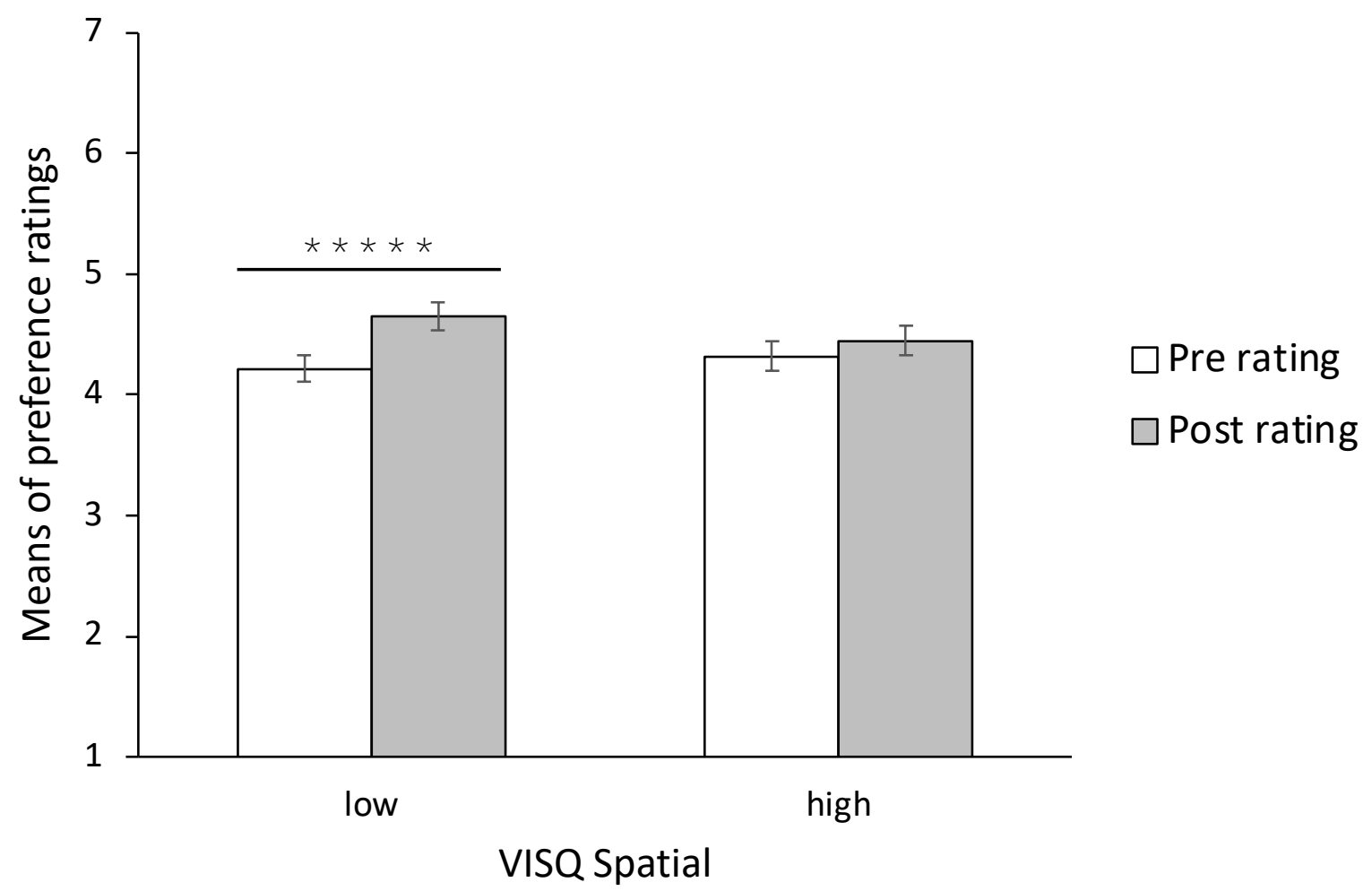

Figure 3: Mean values of the Pre and Post preference ratings for High and Low spatial groups (Experiment 1). ${ }^{* * * * *} p<0.0005$. Error bars show standard error.

Finally, to examine whether and how object visual imagery ability affects the impacts of the image of ownership, a 2 (Group: High or Low object VISQ [hereafter 'High/Low object group'; 16 participants each]) $\times 2$ (Assignment: Self or Other) $\times 2$ (Time of rating: Pre or Post) repeatedmeasures ANOVA was conducted. The results revealed a significant main effect of Time of rating $(F$ $\left.(1,30)=12.321, p=0.001, \eta_{\mathrm{p}}{ }^{2}=0.2911\right)$, and the average preference ratings were higher for Post than 
Pre rating (Figure 4). No other effects were significant.

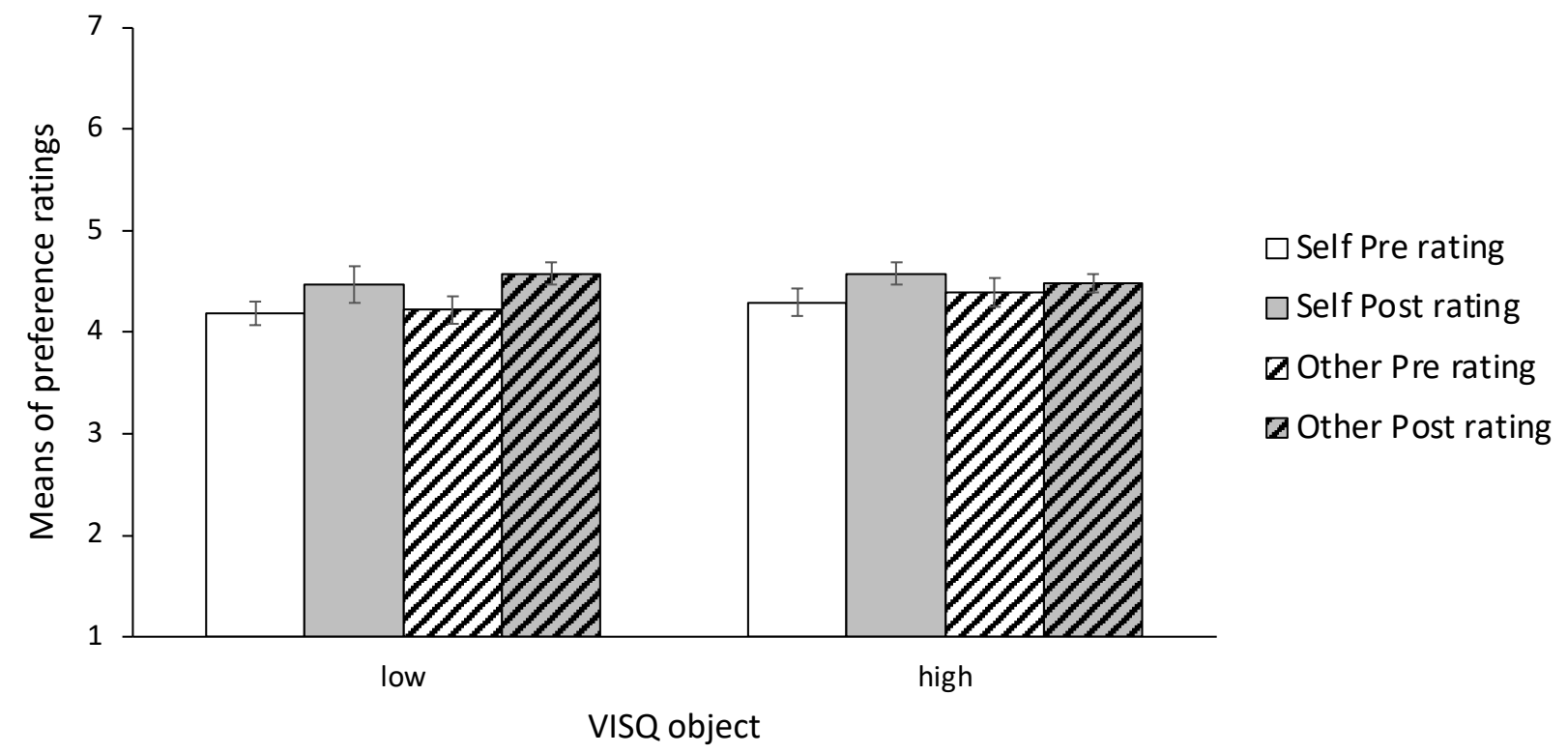

Figure 4: Mean values of the Pre and Post preference ratings in the Self and Other conditions for High and Low object groups (Experiment 1). Error bars show standard error.

\section{Discussion}

In this experiment, participants were provided haptic information about the blocks, so we expected their value to increase in the Self condition and to remain unchanged in the Other condition. In the High total group, the average preference rating increased only in the Self condition. This indicates that when the overall visual imagery ability is high, generating an image of one's ownership of an object about which participants had no prior knowledge but had a haptic experience of increases the value of that object. In contrast, the rating did not increase in the Other condition. This indicates that the value 
of the object does not increase when participants imagine it is owned by another person. Thus, in the High total group, the results were similar to previous findings reported by Kim and Johnson ${ }^{[5]}$.

In the Low total group, the value of the blocks increased only in the Other condition. This suggests that when overall visual imagery ability is low, the value of the object is increased only when one imagines a situation in which others own it. However, given the relatively low imagery ability of this group, it is unlikely that differences regarding who owns the object in the image of ownership will be selectively reflected in the results. Corroborating this, unlike the high total group, the interaction of target $\mathrm{x}$ timing of rating was not significant in the low total group. In addition, when multiple comparisons were attempted, there was a tendency for the average preference ratings to be significantly higher in the Post than Pre condition, even in the Self condition $(F(1,46)=3.415, p=$ 0.071). Considering these results, it seems relatively more reasonable to assume that the value of the object in both the Self and Other conditions was increased by factors that were less related to the image of ownership than to assume that the value of the object was increased only in the Other condition in the Low total group. One of the reasons for this may be that the block was touched. For example, Peck and Shu reported that simply touching an object may increase its perceived value compared to not touching $\mathrm{it}^{[\mathrm{7]}}$. According to them, touching an object increases the psychological ownership and thus the value, because it allows them to manipulate the object freely, as if they owned it in reality. Therefore, in the Low total group, it is likely that only the increase in value caused by touching the object occurred. 
This begs the question: why did the High total group, who also touched the blocks, not show an increase in value in the Other condition? One possibility is that the effect of touching the block was somehow reduced in the Other condition for the High total group. For example, a study by Huang and Wu suggests that negative attitudes towards objects that are imagined to be owned by others (otherderogation) are generated ${ }^{[14]}$. It is likely that such negative attitudes also arose in the Other condition of the High total group, offsetting the effect of touching the block.

In summary, the results suggest that the value of an object is increased by touching it, even if the participant has no prior knowledge of it. On the one hand, when the overall visual imagery ability was low, no imagery effect occurred, suggesting that it was mainly the effect of touching that was reflected in the results. On the other hand, when the total visual imagery ability was high, there was an increase in value in the Self condition, but not in the Other condition. The results for the Other condition suggest that other-derogation ${ }^{[14]}$, a mechanism that lowers the valuation of objects that are not one's own, was at work, lowering the value and thus offsetting the increase in value from touching. The reason the decrease in value caused by the ownership of the object by others occurred only in the High total group may be that its members were able to imagine the situation in which others owned the object relatively more concretely due to their higher imagery ability. It is reasonable to think that the improvement in the value of the Self condition is an effect of imagery rather than an effect of having touched the block.

However, the results of multiple comparisons showed that there was no significant difference 
between the High and Low total groups in the Post preference rating of the Self condition $(t(92)=$ $0.693, p<1)$. This suggests that there was no difference between the Self condition preferences of the High total group, which should have had two facilitators (i.e. touching and visual imagery), and that of the Low total group, which had only one facilitator. Thus, it seems unlikely that the facilitative effects of touching and the image of ownership in the Self condition are cumulative. We speculate that the facilitative effect was saturated by the image factor alone, that is, a ceiling effect occurred.

As described above, the results were as expected only when the overall visual imagery ability was high. This may be because the blocks to be imaged were not seen in daily life, and thus it was relatively difficult to generate mental images.

Next, we discuss the results of grouping by the VISQ subscores. In the Low spatial group, the value of the object was higher in the Post than Pre condition, regardless of the Self and Other conditions. The fact that there was no difference between the Self and Other conditions makes it unlikely that this result is an effect of imagery. This result can be interpreted by considering what spatial imagery ability is represented by the total score in the VISQ spatial: an example of a question about spatial imagery in the VISQ is 'I am good at drawing floor plans and plans of buildings'. Therefore, one aspect of spatial imagery ability could be the ability to grasp spatial attributes such as spatial relationships and arrangements among parts of an object, that is, its structure. Then, the results of the Low spatial group in this experiment can be interpreted as follows. The spatial information of the blocks grasped by the participants in the Low spatial group at the Pre-stage of preference rating was 
less than that of the participants in the High spatial group. As a result, the participants in the Low spatial group must have grasped relatively more spatial information when they touched the block to grasp its structure. This increase in the amount of spatial information about the object may have led to an increase in value.

On the other hand, the High spatial group is presumed to have grasped more spatial information than the Low spatial group in the Pre preference rating because they are thought to have a higher ability to grasp spatial attributes. As a result, there was not much increase in the amount of spatial information by touching the object, which may have prevented the increase in value.

In the analysis in which participants were grouped according to their VISQ object scores, none of the effects involving group factors were significant, suggesting that the degree to which information about the visual appearance of the image, that is, the object's shape, size, color, brightness, and such, can be realistically reproduced, is not involved in the effect of the image of ownership. This may be because, unlike spatial information, visual imagery ability is not necessary to grasp visual information.

The results of grouping according to total VISQ scores were different from those of grouping according to either visual or spatial scores, suggesting that the effect of the image of ownership may not occur if only one of the scores is high, but perhaps if both scores are high. In the previous findings where concrete objects were used as the objects of imagery, the image of ownership had its effect even without grouping as in the present study ${ }^{[5]}$. Presumably, if the object of the image is a concrete 
thing, it is easy to imagine the object, and therefore, the image of ownership may have had an effect regardless of the level of imagery ability.

If the results observed in the High total group, in which value increased in the Self condition but not in the Other condition, were due to recall of the haptic experience of the blocks, then these results could not have been obtained without the experience of touching the blocks. Similarly, if the increase in value observed only for participants with lower ability to grasp spatial attributes was due to touching the blocks, this result could also not have been obtained without the experience of touching the blocks. Therefore, in Experiment 2, we asked participants to form images of their own and others' ownership without letting them touch the blocks, which were the target of the preference rating, to determine if the results of Experiment 1 could be reproduced.

\section{Experiment 2}

\section{Results}

ANOVAs for the same factorial design as in Experiment 1 were conducted. First, to examine whether and how overall visual imagery ability affects the impact of the image of ownership, a 2 (Group: High or Low total [16 participants each]) $\times 2$ (Assignment: Self or Other) $\times 2$ (Time of rating: Pre or Post) repeated-measures ANOVA was conducted. The results revealed a significant main effect of Time of rating $\left(F(1,30)=10.749, p=0.003, \eta_{\mathrm{p}}{ }^{2}=0.264\right)$. No other effects were significant (Figure 5a). 
a

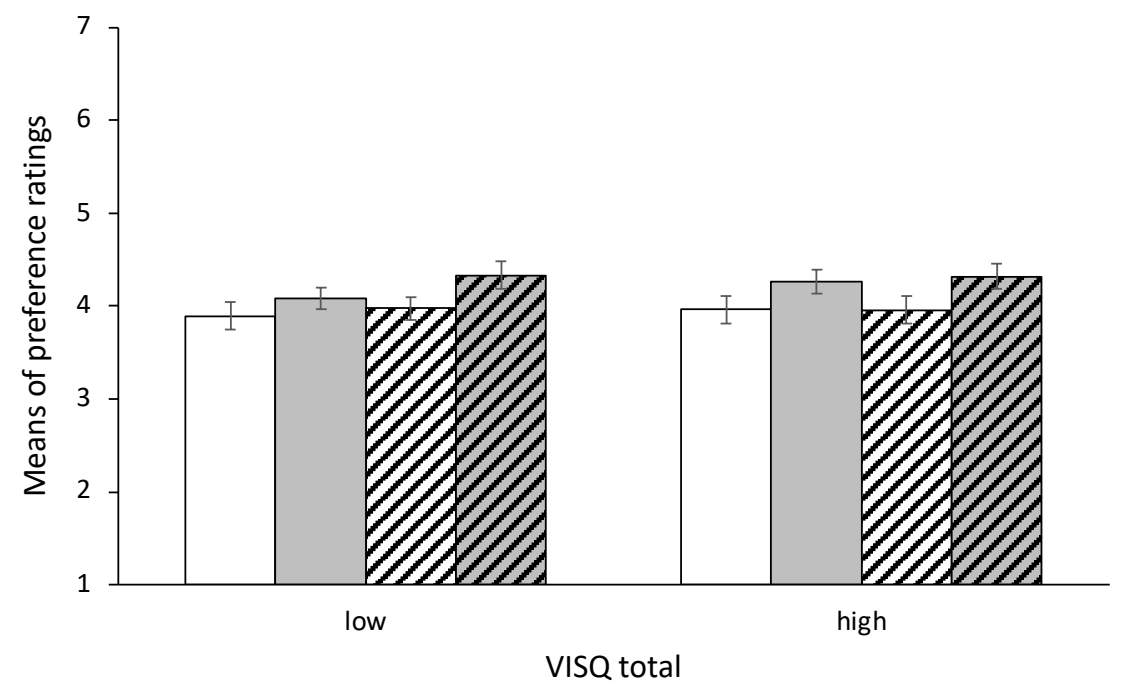

$\square$ Self Pre rating

$\square$ Self Post rating

$\square$ Other Pre rating

$\square$ Other Post rating

b

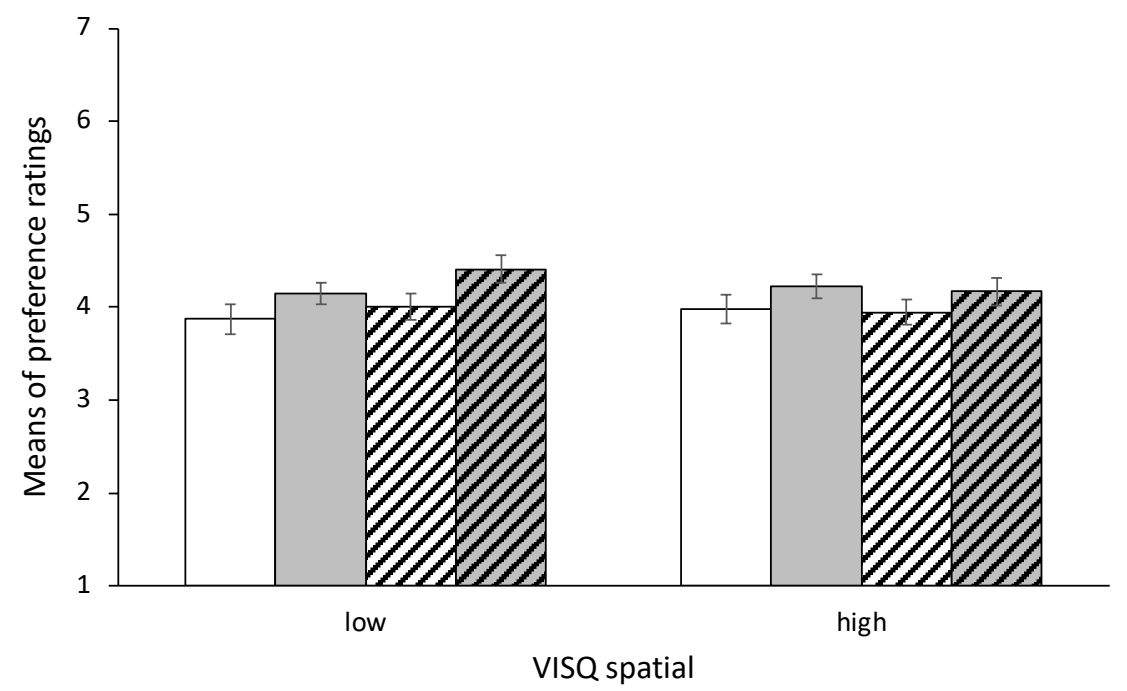

$\square$ Self Pre rating

$\square$ Self Post rating

$\square$ Other Pre rating

$\square$ Other Post rating

C

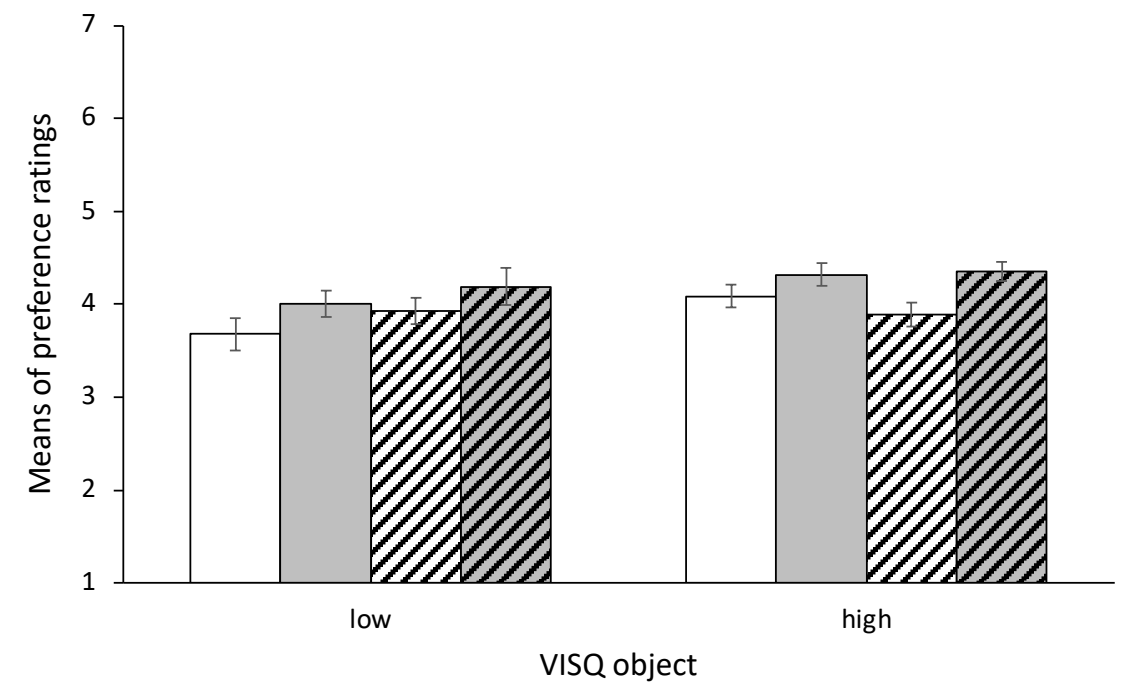

$\square$ Self Pre rating

$\square$ Self Post rating

$\square$ Other Pre rating

[D Other Post rating 
Figure 5: Mean values of the Pre and Post preference ratings in the Self and Other conditions for

(a)High and Low total groups, (b)High and Low spatial groups, (c)High and Low object groups

(Experiment 2). Error bars show standard error.

Next, to examine whether and how spatial-visual imagery ability affects the impacts of the image of ownership, a 2 (Group: High or Low spatial [16 and 14 participants, respectively]) $\times 2$ (Assignment: Self or Other) $\times 2$ (Time of rating: Pre or Post) repeated-measures ANOVA was conducted. There was a significant main effect of Time of rating $\left(F(1,28)=7.794, p=0.009, \eta_{\mathrm{p}}{ }^{2}=0.218\right)$. Again, no other significant effects were observed (Figure 5b).

Finally, to examine whether and how object visual imagery ability affects the impacts of the image of ownership, we conducted a 2 (Group: High or Low object [16 and 13 participants, respectively]) $\times$ 2 (Assignment: Self or Other) $\times 2$ (Time of rating: Pre or Post) repeated-measures ANOVA. There was a significant main effect of Time of rating $\left(F(1,27)=10.704, p=0.003, \eta_{\mathrm{p}}{ }^{2}=0.284\right)$. No other effects were significant (Figure 5c).

\section{Discussion}

In this experiment, we did not provide participants any tactile information about the blocks, so we expected no change in value in either the Self or Other condition. In contrast to Experiment 1, no significant effects involving group factors were detected, regardless of how the groups were classified 
according to their VISQ scores; and in all analyses, only the main effect of the Time of rating was

significant. Thus, it is reasonable to assume that neither the image of ownership nor individual differences in mental imagery affected the results, and that seeing each block twice induced the mere exposure effect.

\section{General Discussion}

The present study tested whether the presence or absence of haptic information about an object affects the increase in the value of that object due to the image of ownership. In Experiment 1, in which participants touched the blocks and were provided haptic information, it was expected that the value of the blocks would increase when they were associated with the self, but not with others. In Experiment 2, in which participants were not allowed to touch the blocks, the value was not expected to increase in either condition. The results of Experiment 1 showed that in the High total group, the Post preference rating was significantly higher in the Self condition, while it did not change in the Other condition. In the Low total group, the Post preference rating tended to be significantly higher in both the Self and Other conditions. Additionally, in the Low spatial group, the results of the analysis that collapsed across the Self and Other conditions showed that the Post preference rating was significantly higher regardless of the subject (Self/Other) participants associated the block with. In the High spatial group, there was no change in the preference rating between Pre and Post conditions.

The results of Experiment 2 showed that, unlike in Experiment 1, the group factors showed no 
effect, and only the main effect of Time of rating was significant: The Post preference ratings were higher than that of the Pre preference.

The results of the High total group in Experiment 1 suggest that when the overall visual imagery ability is high, the value increases when the object is associated with the self, but it does not change when it is associated with others. On the other hand, the results of the Low total group suggested an increase in value in both the Self and Other conditions. A relatively low imagery ability would probably result only in a mere exposure effect. A similar effect was observed in all groups in Experiment 2. Thus, if the participant has no haptic experience of the object, only the mere exposure effect would occur.

Although the mere exposure effect should have also occurred in the High total group in Experiment 1 , there was no increase in value in the Other condition. This result can be interpreted as follows. The High total group would have been able to form a concrete image of ownership in the Other condition as well as in the Self condition. As a result, they could recall almost the same amount of haptic information in the Other condition as in the Self condition. Therefore, in the Other condition of this group, the haptic images of objects belonging to another person became relatively more realistic, which may have caused stronger other-derogation. This would have decreased the value of the object and offset the mere exposure effect.

Based on the results of Experiments 1 and 2, it seems that the effects of the image of ownership found in the High total group in Experiment 1 can only be obtained when the object to be owned has 
been touched beforehand. Therefore, as expected, this suggests that the association between the self and an object gained through the image of ownership is caused by recalling haptic information about that object. It is possible that participants in the High total group could produce a more realistic image of owning the object, and thus were able to recall more haptic information about the object as part of their image of it.

Why does the recall of haptic information about an object create an association between the self (and/or the other) and the object? If we take the expression 'become part of (an) extended self ${ }^{[2,11]}$ literally, it may mean that the integration of one's own body representation and that of the object is (temporarily) enhanced. For example, Iriki, Tanaka, and Iwamura showed that the active use of tools can integrate body representations with those of tools ${ }^{[15]}$. Iriki et al. used Japanese monkeys as test subjects to investigate how the visual receptive field changes before and after the use of a tool (rake). The results showed that the receptive field extends to the periphery of the tool. This suggests that the participants' body representations were extended to the area around the tool. The use of tools naturally leads to the acquisition of haptic information about these objects. It is possible that this expansion of body representation occurred as a result of the recall of haptic information when generating an image of ownership, but the results of this study do not allow us to examine whether this is true. If we could compare receptive fields as in Iriki's study, we might find that the receptive fields for items associated with the participants themselves are relatively more widely distributed than those for items associated with others. 
The analysis of grouping by spatial VISQ scores in Experiment 1 suggests that grasping spatial information about an object increased its value. Considering that the same result was not obtained in Experiment 2, in which the participants did not touch the blocks, the spatial information obtained by touching the blocks contributed to the result. At the beginning of the main trials of the association task in Experiment 1, participants knew that they would touch the block before forming an image of ownership. Therefore, it is possible that they were touching it so that they could inspect its details closely to create an accurate image of the block. It is unclear at this point whether such intentional 'exploring' is essential for the enhancement of value.

According to Lederman and Klatzky, spatial information is first roughly grasped by sight and then populated with elaborate information using haptic sense ${ }^{[16]}$. The same process may have been at work in this study when the participants touched the blocks with their hands. However, spatial information can also be obtained by observing the blocks from various directions. It is unclear whether the method of grasping spatial information that improves value is limited to the haptics, and if so, why. This issue should be examined in future studies.

What kind of information, among all that is obtained through haptic sense, caused the value to increase? In this study, the participants touched six sample blocks to familiarise themselves with the objects. Therefore, we can assume that the value was enhanced by obtaining information about the structure of each block touched in the association phase, such as size, shape, and the position of the centre of gravity, rather than information about the surface such as texture and hardness which are 
common to all blocks.

Therefore, in the Low spatial group in Experiment 1, it is presumed that information about the structure of the block increased the value. Why then does obtaining information about the structure increase the value? Information about the structure is obtained by grasping the spatial arrangement of the elements of the object and understanding the whole picture. Muth and Carbon showed that understanding the whole picture of an object can lead to a kind of 'Aha experience', which may increase its value ${ }^{[17]}$. For the participants in the Low spatial group in Experiment 1, noticing the structure of the block by touching it with their hands may have brought about a pleasure similar to the 'Aha experience' which led to an increase in value. It is speculated that the participants in the Low spatial group in Experiment 1 obtained information about the structure by touching the blocks. In the future, we could verify this by quantitatively measuring the amount of structural information that the participants obtained before and after touching.

In conclusion, the present study suggests that the enhancement of the value of an object associated with imagining owning the object occurs when the image can be formed realistically and when haptic information about the object is possessed. In addition, the findings indicate that the value of an object is enhanced when spatial information about the object which cannot be completely grasped through vision is gleaned through the haptics. Previous research on value perception has rarely measured individual differences such as the vividness of mental images. Future research can clarify the mechanism by which people perceive value in more detail by considering individual differences in 
visuospatial (imagery) ability.

\section{Method}

\section{Experiment 1}

\section{Participants}

Twenty females and 20 males (mean age $=21.7$ years old, $\mathrm{SD}=2.87$ years old) voluntarily participated in exchange for payment. They were all right-handed and had a normal or corrected-tonormal vision (soft contact lenses or glasses). This study was approved by the Research Ethics Committee of Hiroshima University (approval number E-965-6). In addition, all experiments in this study were conducted in accordance with the ethical standards and guidelines of the Declaration of Helsinki. All participants received an explanation of this study and provided informed written consent before participating in this study.

\section{Stimuli and apparatus}

A total of 34 blocks consisting of multiple 'arms' (linearly connected cubes) were used. All the blocks were made by gluing polyacetal cubes that were $2 \mathrm{~cm}$ on each side and chamfered by $0.5 \mathrm{~mm}$. The blocks were painted matte white and had a uniform texture. They were made of nine or ten cubes. Twenty-eight were used for preference ratings, and the remaining six were used for familiarisation (see below). 
The 28 blocks for the main experiment were divided into two groups of 14 blocks each so that the preferences rated in the pilot study and structure attributes were uniform. The attributes of the blocks were the number of cubes, arms, and planes formed by the two arms (Figure 6). Six participants participated in the pilot study. None of these participants took part in the main experiments reported in this study. They rated their preferences in the same way as the participants in the main experiment (described below).

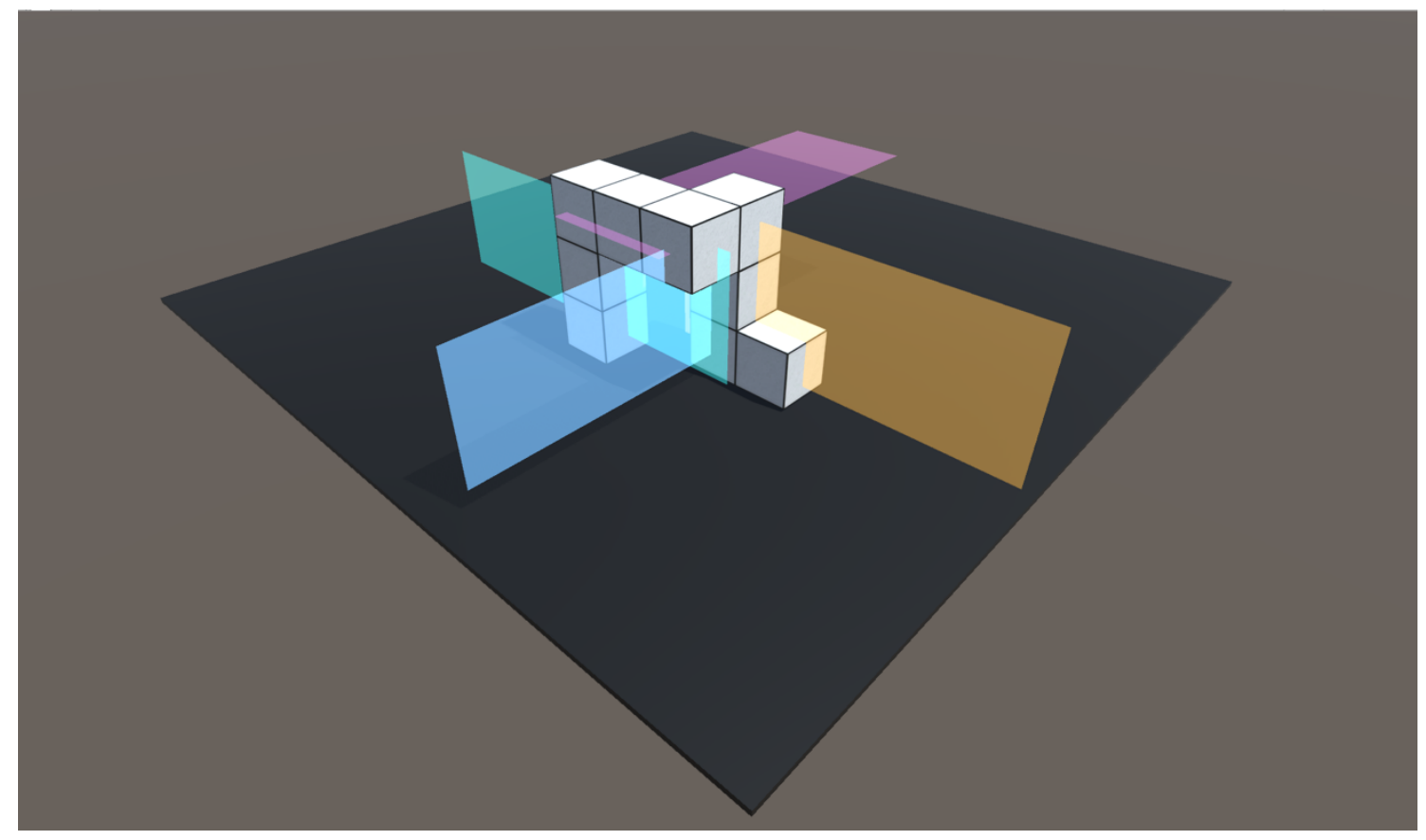

Figure 6: Planes formed by the two arms. This block has nine cubes, five arms, and four planes

The attributes of the block groups used for preference ratings are shown in Supplementary

Appendix 1. The appearances of the blocks for both groups are shown in Supplementary Appendices 2 and 3. 
After the pilot experiment was completed, it was found that one of the blocks did not meet the above conditions because it had only two planes. Therefore, we replaced this block with one that had the same number of cubes and arms but had three planes. The data obtained from the replaced blocks were excluded from the analysis.

A t-test was conducted on the preference ratings obtained in the pilot experiment to verify whether the preferences of the two block groups were equivalent. A corresponding t-test was performed on the preferences of the two groups, and no significant differences were detected $(t(15)=0.222, p<1)$. Therefore, we considered the preference ratings of the two block groups to be nearly equal. The only purpose of creating two groups of blocks was to allow for variations in the stimuli. Each group was randomly assigned to one of the 20 participants.

The attributes of the six blocks used for familiarisation are presented in Supplementary Appendix 4. The appearances of the blocks are presented in Supplementary Appendix 5.

The blocks were placed on a setting board covered with black corduroy cloth, and the size of the setting board was $35 \times 42 \mathrm{~cm}$.

The VISQ ${ }^{[12]}$ is a self-report questionnaire that measures object imagery and spatial imagery abilities. These abilities are related to object and spatial factors as derived from the OSIQ ${ }^{[13]}$. In the VISQ, both object and spatial imagery abilities are measured using 12 questions on a 5-point scale. The internal consistency of the VISQ is $\alpha=0.870$ for the object imagery scale and $\alpha=0.846$ for the spatial imagery scale, indicating an appropriate level. The test-retest reliabilities at 1-month interval 
are also at an appropriate level, $r=0.751$ for the object imagery scale and $r=0.831$ for the spatial imagery scale.

Procedure

The experiment was conducted in a soundproof room for each participant. In the soundproof room, there was a desk, a chair for the participants to sit on, and a setting board on the desk (Figure 7). The distance from the participant's chair to the centre of the setting board was approximately $70 \mathrm{~cm}$. 


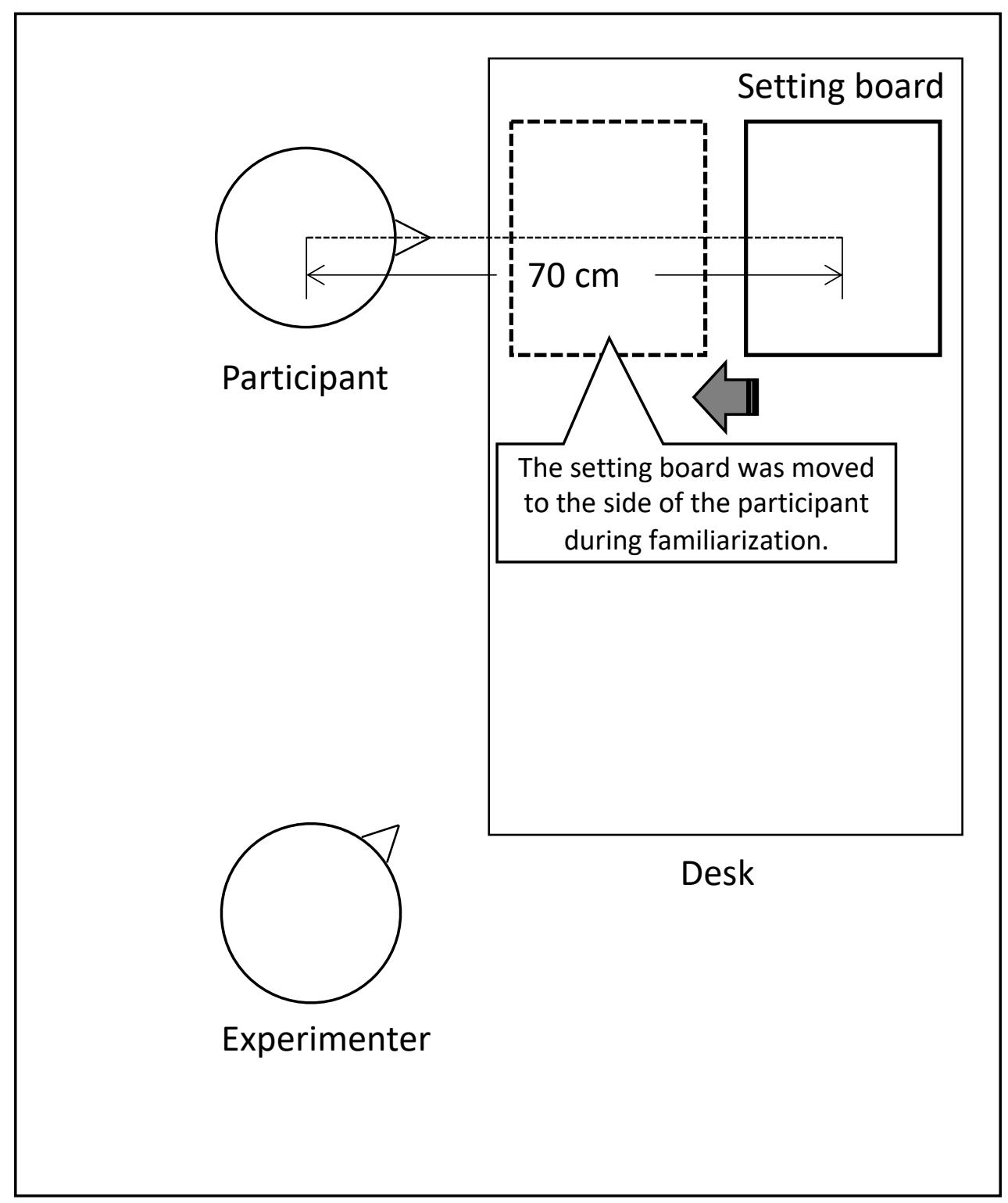

Soundproof room

Figure 7: Experimental setting

The experiment consisted of five tasks: the Pre preference rating, familiarisation, association

(physically grasping the block and generating a mental image of owning it), answering the questionnaire (VISQ), and the Post preference rating. These tasks were performed in the described order (Figure 8). 


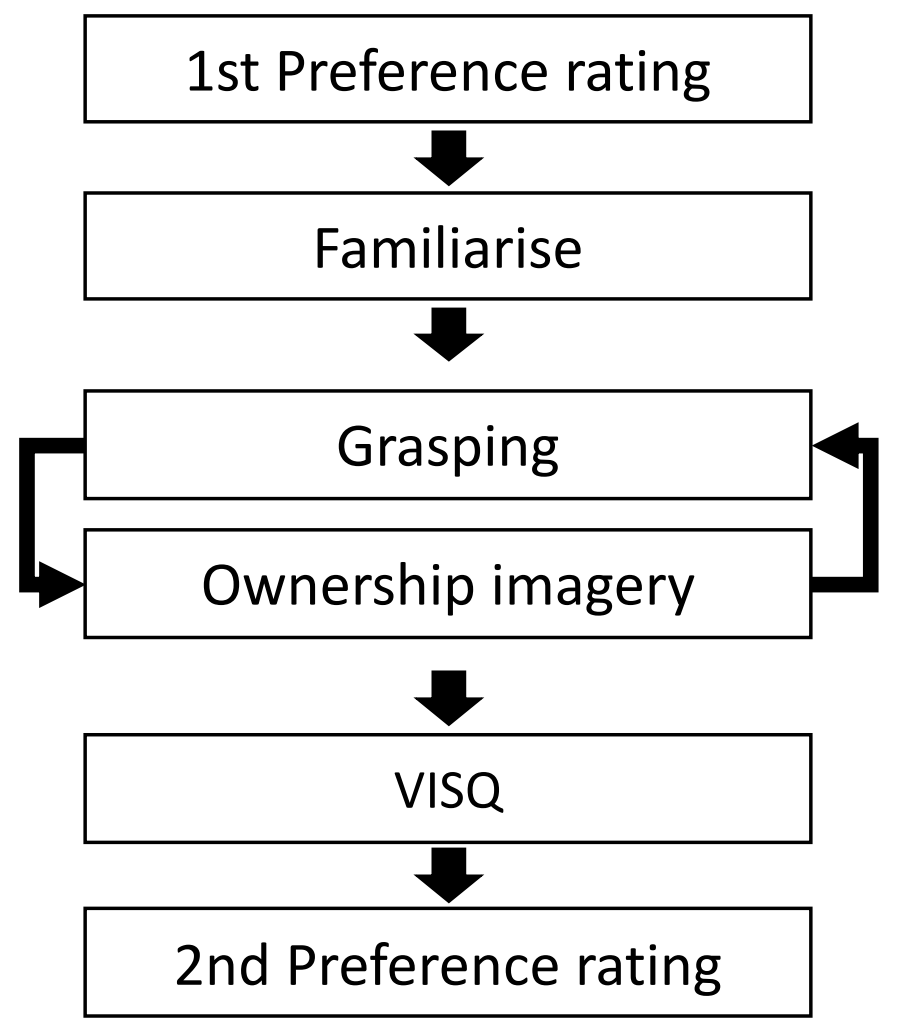

Figure 8: The tasks performed in the experiment and their order.

In the Pre preference rating, participants rated their preferences for the blocks on a 7-point scale,

which were presented one at a time in random order. This task began by asking the participants to close their eyes. After the experimenter placed one block on the board, the participants were asked to open their eyes. Each block was placed at the opposite end of the setting board from the participant. Participants were asked to respond to their preferences without thinking too much.

To record their preference, participants were provided with an A5 size booklet with a stimulus number (e.g. 'Stimulus 1'), a 7-point scale, and a phrase describing the scale (1: very much dislike to 
7: very much like) on each page (Supplementary Appendix 6). It was explained verbally that the rating value of 4 corresponded to 'neither'. Participants were asked to circle the number corresponding to their perceived degree of preference.

Familiarisation was then conducted. Participants were asked to freely touch all six blocks on the setting board and to continue touching for five minutes. The board was positioned near the participant.

The association task was then conducted in which the board was returned to its original position, and two trays were set up between the board and the participant. Each tray had a piece of paper labelled 'you' or 'experimenter' on it (in Japanese). It was counterbalanced among the participants as to whether the 'you' or 'experimenter' tray was placed on the left or right side as seen by the participant. The visual mental image was then induced as follows. 'To imagine is to picture a scene in your mind as if you were seeing it. For example, when you remember something from the past, you may see an image or movie in your mind. That is visual mental image'.

The procedure of the association task was demonstrated through practice trials. First, the participants were asked to close their eyes. Next, two of the blocks used during familiarisation were placed on the participant's side of the board. The blocks used for instruction and their placement were always the same in the two experiments reported in this paper (No. 1 and No. 2 in Supplementary Appendix 5). After the experimenter had finished arranging the blocks, the participants were asked to open their eyes. Participants were first asked to pick up the left block and touch it for 30 seconds to grasp its structure. They were then asked to perform the same with the right block. After touching 
each block, they were asked to return it to the original place. Next, participants were asked to form an image of a scene in which the block became the participant or experimenter's property (image of ownership). First, the participant was told that the block on the right belonged to the participant, and the participant was asked to move it to the tray with the 'you' label on it. They were then asked to close their eyes for 10 seconds and form an image of a scene in which they owned the block. For the block on the left, they were told that it belonged to the experimenter, and they were asked to place it on the tray labelled 'experimenter'. They were then asked to close their eyes for 10 seconds and form an image of a scene in which the experimenter owned the block. After these instructions and practice trials, the main trial was conducted using the same procedure for the blocks for which the participant rated their preferences. In the main trials, blocks with the same attributes (number of cubes, arms, and planes) were paired, and the pairs were fixed throughout the experiment. The order in which each pair of blocks was presented, the order in which they were held, and whether they were assigned to the participant or experimenter were randomised across participants.

The participants then answered a questionnaire (VISQ). After that, the Post preference rating was conducted in the same way as the Pre preference rating.

\section{Data analyses}

The factors of the two stimulus groups were not included in the analysis because they were not of interest in this study. Data from participants whose preference ratings were outliers (above or below 
$\mathrm{M} \pm 2 \mathrm{SD}$ ) were excluded, as were data from participants whose total for each VISQ score (object,

spatial, total [object + spatial]) was outlier. This excluded the data of eight participants. Participants were then grouped according to whether they were above or below the median of each VISQ score.

Participants who fell into the median were excluded from the analysis. ANOVA4 on the Web (https://www.hju.ac.jp/ kiriki/anova4/index_js.html) was used for analysis of variance. Ryan’s method was used for multiple comparisons of analyses of variance.

When grouped according to their VISQ total score, six participants who fell into the median were excluded. As a result, 11 participants in the High total group and 14 in the Low total group were included in the analysis. When grouped according to their VISQ spatial scores, three participants who fell into the median were excluded. As a result, 14 participants in the High spatial group and 15 in the Low spatial group were included in the analysis. When grouped according to their VISQ object scores, no participants fell into the median. As a result, 16 participants in both the High and Low object groups were included in the analysis.

\section{Experiment 2}

\section{Participants}

Forty participants who had not participated in either the pilot experiment or Experiment 1 participated in Experiment 2. Twenty-three women and 17 men (mean age $=22.1$ years old, $\mathrm{SD}=2.82$ years old) voluntarily participated in exchange for payment. They were all right-handed and had a normal or 
corrected-to-normal vision (soft contact lenses or glasses). Other aspects were the same as in Experiment 1.

Stimuli

The same blocks as in Experiment 1 were used.

Procedure

The procedure followed Experiment 1, except that there was no opportunity to handle the object in the association task; the participants did not touch any blocks.

\section{Data analyses}

The methods of data analysis and the exclusion criteria for the participants were the same as those in

Experiment 1. Data from six participants who fell into outliers were excluded. When grouped according to their VISQ total score, two participants who fell into the median were excluded. As a result, 16 participants in both the High and Low total groups were included in the analysis. When grouped according to their VISQ spatial scores, four participants who fell into the median were excluded. As a result, 16 participants in the High spatial group and 14 in the Low spatial group were included in the analysis. When grouped according to their VISQ object scores, five participants who fell into the median were excluded. As a result, 16 participants in the High object group and 13 in the 
Low object group were included in the analysis.

\section{Acknowledgements}

We are deeply grateful to Dr. Tokiko Harada for her helpful comments on the early planning of the

experiment.

This work was supported by the Japanese Science and Technology (JST) agency Center of Innovation

(COI) grant number JPMJCE1311.

We would like to thank Editage (www.editage.com) for English language editing.

\section{Author contribution statement}

K.H. and T.S. designed the study with advice from K.O. and S. Y.; K.H. conducted the experiments and analysed the data. K.H. drafted the first version of the manuscript. All authors reviewed the manuscript and approved the final version of the manuscript.

\section{Competing interests}

The authors declare no competing interests.

\section{Data availability}

The datasets generated during and/or analysed during the current study are available from the 
corresponding author on reasonable request.

\section{References}

1. Beggan, J. K. On the social nature of nonsocial perception: the mere ownership effect. J. Pers. Soc. Psychol. 62, 229-237 (1992). 10.1037/0022-3514.62.2.229.

2. De Dreu, C. K. \& van Knippenberg, D. The possessive self as a barrier to conflict resolution: effects of mere ownership, process accountability, and self-concept clarity on competitive cognitions and behavior. J. Pers. Soc. Psychol. 89, 345-357 (2005). 10.1037/0022-3514.89.3.345, Pubmed:16248718.

3. Cunningham, S. J., Turk, D. J., Macdonald, L. M. \& Neil Macrae, C. N. Yours or mine? Ownership and memory. Conscious. Cogn. 17, 312-318 (2008). 10.1016/j.concog.2007.04.003, Pubmed:17543540.

4. Sui, J., Rotshtein, P. \& Humphreys, G. W. Coupling social attention to the self forms a network for personal significance. Proc. Natl. Acad. Sci. U. S. A. 110, 7607-7612 (2013). 10.1073/pnas.1221862110, Pubmed:23610386.

5. Kim, K. \& Johnson, M. K. Extended self: medial prefrontal activity during transient association of self and objects. Soc. Cogn. Affect. Neurosci. 7, 199-207 (2012). 10.1093/scan/nsq096, Pubmed:21148177.

6. Pierce, J. L., Kostova, T. \& Dirks, K. T. The state of psychological ownership: integrating and extending a century of research. Rev. Gen. Psychol. 7, 84-107 (2003). 10.1037/1089-2680.7.1.84.

7. Peck, J. \& Shu, S. B. The effect of mere touch on perceived ownership. J. Consum. Res. 36, 434-447 (2009). 10.1086/598614.

8. Iseki, S. \& Kitagami, S. Mere touching imagery promotes purchase intention through increased psychological ownership. JHES. (in Japanese) 14, 49-54 (2016). 10.4189/shes.14.49.

9. Peck, J., Barger, V. A. \& Webb, A. In search of a surrogate for touch: the effect of haptic imagery on perceived ownership. J. Consum. Psychol. 23, 189-196 (2013). 10.1016/j.jcps.2012.09.001.

10. McKelvie, S. J. The VVIQ as a psychometric test of individual differences in visual imagery vividness: a critical quantitative review and plea for direction. J. Ment. Imag. 19, 1-106 (1995). 
11. McAvinue, L. P. \& Robertson, I. H. Measuring visual imagery ability: a review. Imagin. Cogn. Personal. 26, 191-211 (2007). 10.2190/3515-8169-24J8-7157.

12. Kawahara, M. \& Matsuoka, K. Development of the visual imagery style questionnaire. Jpn. J. Ment. Imag. (in Japanese) 7, 19-31 (2009).

13. Blajenkova, O., Kozhevnikov, M. \& Motes, M. A. Object-spatial imagery: a new self-report imagery questionnaire. Appl. Cognit. Psychol. 20, 239-263 (2006). 10.1002/acp.1182.

14. Huang, Y. \& Wu, Y. Ownership effect can be a result of other-derogation: evidence from behavioral and electrophysiological studies. PLOS ONE 11, e0166054 (2016). 10.1371/journal.pone.0166054, Pubmed:27814395.

15. Iriki, A., Tanaka, M. \& Iwamura, Y. Coding of modified body schema during tool use by macaque postcentral neurones. NeuroReport 7, 2325-2330 (1996). 10.1097/00001756-199610020-00010, Pubmed:8951846.

16. Lederman, S. J. \& Klatzky, R. L. Haptic perception: a tutorial. Atten. Percept. Psychophys. 71, 14391459 (2009). 10.3758/APP.71.7.1439, Pubmed:19801605.

17. Muth, C. \& Carbon, C. C. The aesthetic aha: on the pleasure of having insights into Gestalt. Acta Psychol. 144, 25-30 (2013). 10.1016/j.actpsy.2013.05.001, Pubmed:23743342.

\section{Figure legends}

Figure 1: An example of the blocks used in this study.

Figure 2: Mean values of the Pre and Post preference ratings in the Self and Other conditions for High and Low total groups (Experiment 1$){ }^{*} p<0.05,{ }^{* * *} p<0.005$. Error bars show standard error.

Figure 3: Mean values of the Pre and Post preference ratings for High and Low spatial groups

(Experiment 1). ${ }^{* * * * *} p<0.0005$. Error bars show standard error.

Figure 4: Mean values of the Pre and Post preference ratings in the Self and Other conditions for High 
and Low object groups (Experiment 1). Error bars show standard error.

Figure 5: Mean values of the Pre and Post preference ratings in the Self and Other conditions for (a)High and Low total groups, (b)High and Low spatial groups, (c)High and Low object groups (Experiment 2). Error bars show standard error.

Figure 6: Planes formed by the two arms. This block has nine cubes, five arms, and four planes.

Figure 7: Experimental setting.

Figure 8: The tasks performed in the experiment and their order. 